\title{
Detached-Leaflet Evaluation of Tomato Germplasm for Late Blight Resistance and Its Correspondence to Field and Greenhouse Screenings
}

Majid R. Foolad, Department of Plant Science and the Intercollege Graduate Degree Programs in Plant Biology and MCIBS, The Pennsylvania
State University, University Park 16802; Matthew T. Sullenberger, Department of Plant Science and the Intercollege Graduate Degree Pro-
grams in Plant Biology, The Pennsylvania State University; and Hamid Ashrafi, Department of Plant Science, The Pennsylvania State Urams in

\begin{abstract}
Foolad, M. R., Sullenberger, M. T., and Ashrafi, H. 2015. Detached-leaflet evaluation of tomato germplasm for late blight resistance and its correspondence to field and greenhouse screenings. Plant Dis. 99:718-722.

Breeding for disease resistance requires efficient techniques for screening large plant populations. Late blight (LB), caused by the oomycete Phytophthora infestans, is one of the most devastating diseases of tomato (Solanum lycopersicum) worldwide, and there is a great interest in developing cultivars with resistance to this pathogen. Screening for LB resistance is commonly conducted under field or greenhouse conditions using whole plants. In a previous study, we demonstrated correspondence between field and greenhouse screening of tomato for LB resistance. Here, we report the use of a detached-leaflet assay for such screening. Seventytwo genotypes from two tomato species, varying in degree of resistance and susceptibility to LB, were evaluated in two replicated experiments

for response to LB in a detached-leaflet assay, and the results were compared with those previously obtained from field and greenhouse screening of the same genotypes. There were significant $(P<0.001)$ positive correlations between replications (average $r=0.75$ ) and experiments (average $r=0.72$ ), suggesting that the detached-leaflet experiments were consistent. Further, there were significant $(P<0.001)$ positive correlations between responses in the detached-leaflet assay and those from field $(r=0.82)$ and greenhouse screenings $(r=0.84)$, suggesting reliability of the detached-leaflet assay. The results indicate the utility of the detachedleaflet assay for evaluating tomato for LB resistance, which may facilitate screening of large breeding populations.
\end{abstract}

Late blight (LB), caused by the oomycete Phytophthora infestans (Mont.) de Bary, is a destructive disease of the cultivated tomato (Solanum lycopersicum L.) and potato (S. tuberosum L.), with the potential to quickly destroy all aboveground plant organs as well as potato tubers. If crops are unprotected, LB can destroy an entire tomato or potato crop within 7 to 10 days $(3,16)$. Economic losses due to LB can include reduced yield, lower fruit quality, diminished storability, and costs associated with fungicide application (16). Most commercial cultivars of tomato are susceptible to $\mathrm{LB}$, and the disease is currently controlled mainly by cultural practices and frequent use of fungicides. Recently, there have been intense efforts to develop tomato cultivars with improved resistance to LB, and a few resistant cultivars have been released $(5,6,17)$ (http://www.johnnyseeds.com/ p-8473-defiant-phr-f1.aspx; http://www.highmowingseeds.com/OrganicIron-Lady-F1-Hybrid-Tomato-Seeds.html).

Any project that aims to improve genetic resistance in breeding material requires accurate and efficient methods of disease screening. Screening for LB resistance is typically conducted under field or greenhouse $(\mathrm{GH})$ conditions using whole plants. These methods have advantages and disadvantages. Field screening provides the opportunity for evaluating large populations under production conditions with either natural or artificial inoculum. Potential drawbacks to largescale field screening include reduced uniformity of disease pressure and confounding effects due to uncontrollable environmental and biological factors (e.g., other pathogens). GH evaluations may allow screening for resistance to isolates that have not been found in the field, though great caution must be taken to prevent spread of the

Current address of H. Ashrafi: Department of Plant Sciences, Seed Biotechnology Center, University of California, Davis, CA 95616.

Corresponding author: M. R. Foolad; E-mail: mrf5@psu.edu

Accepted for publication 19 November 2014.

http://dx.doi.org/10.1094/PDIS-08-14-0794-RE

(C) 2015 The American Phytopathological Society pathogen outside of the GH. Conducting evaluations in the GH allows for precise control of environmental conditions favorable for inoculation, disease infection, and progression. These evaluations are also independent of potential confounding factors such as plant growth habit, fruit load, and other disease agents. However, the cost and availability of confined $\mathrm{GH}$ facilities may limit their use.

Screening for LB resistance may also be conducted using detached leaves or leaflets incubated in a growth chamber. The detachedleaflet assay has been used to evaluate for resistance to LB since the 1950 s, mainly in potato $(2,10,13-15,19,22,23)$. Leaflets are inoculated by either dipping a leaflet in a suspension of $P$. infestans or dropping inoculum on the abaxial (lower) leaflet's surface using a micropipettor, followed by incubation under favorable conditions for pathogen infection, development, and establishment (see Materials and Methods). Leaflets should be of similar physiological age for reliable results. For evaluation, lesion size, rate of lesion expansion, infection efficiency, latency period, spore density (1), and fungal biomass (9) can be used as indicators of resistance to LB. Evaluations using detached leaflets offer several practical advantages, including minimal requirement of space and facilities, limited risk of pathogen escape, and rapid screening of large breeding populations. However, there have been mixed reports of the reliability of this screening technique and its correlation with field or GH evaluations. Several studies reported good correlations between screening results from detachedleaflet assays and those obtained from field or GH trials $(7,10,11,19,24)$. A few studies did not always find clear correlations between field resistance and disease response in the detached leaf or leaflet assays. For example, Dorrance and Iglis (2) observed variation within and between detached-leaflet experiments, and Malcolmson (14) reported that the main components of susceptibility, amount of infection, lesion size, and sporulation were not always consistent in the detached-leaflet experiments. The latter author, however, found "close correlation between the assessment of resistance obtained from detached leaflets and from whole plants in the laboratory and in the field".

No formal study has been conducted to determine the accuracy and reliability of the detached-leaflet assay for screening tomato for LB resistance, and it is not known whether resistance observed at this level reflects the resistance observed under field or GH conditions. 
Without accurate, reliable, and efficient disease screening assays, the presence of true genetic resistance cannot be ascertained with confidence. In a recent study, we reported the response of 67 accessions of tomato wild species $S$. pimpinellifolium $\mathrm{L}$. and 5 S. lycopersicum control genotypes to LB and compared results of GH screening with field screening (4). It was determined that, across all accessions, there was a good correspondence between response to LB under field and GH conditions, suggesting that GH experiments could be used reliably to screen tomato breeding germplasm for response to LB. The present study was undertaken to determine the reliability of detached-leaflet assay for screening tomato for LB resistance, and to determine whether resistance in the detached-leaflet assay reflects the resistance observed under field or GH conditions.

\section{Materials and Methods}

Plant material. Sixty-seven accessions of the tomato wild species $S$. pimpinellifolium and five $S$. lycopersicum control genotypes were used in this study (Table 1). Original seed of the wild accessions were obtained from the United States Department of Agriculture (USDA) Agricultural Research Service Plant Genetic Resources Unit (PGRU), Geneva, NY (plant introduction [PI] numbers) and the C. M. Rick Tomato Genetics Resource Center (TGRC), University of California, Davis (LA numbers). Seed of the control genotypes were received from R. G. Gardner, North Carolina State University, Mills River, NC.

Inoculum preparation. An aggressive $P$. infestans isolate (PDA8030275, also known as Rock Springs [RS]-2004), belonging to U.S. clonal lineage US-13, mating type A2, and race/genotype T-1, was originally obtained from S. Kim at the Pennsylvania Department of Agriculture. This isolate was chosen because of its aggressiveness on tomato and presence in the local area at the time of these experiments. Inoculum preparation was as reported elsewhere (4). The sporangial concentration before inoculation was adjusted to approximately 20,000 sporangia/ml.

Detached-leaflet screening experiments. Two detached-leaflet screening experiments (designated I and II) were conducted at different times. In each experiment, seedlings of each genotype were grown in 72-cell seedling flats in the GH. When seedlings were 4 to 6 weeks old, six newly expanded leaflets from the third or fourth leaf of each genotype were detached and used for the experiment (equivalent to six replications per genotype per experiment). The detached leaflets were placed upside down on the lids of two sterile 9-cm petri dishes (three leaflets per petri dish) containing a thin layer of $1.7 \%$ water agar. Each leaflet was inoculated by placing three $10-\mu 1$ droplets of $P$. infestans inoculum (each with approximately 200 sporangia) on the lower surface of the leaflet. In total, 18 droplets (six leaflets, each with 3 droplets) were employed per genotype per experiment. To maintain high humidity, the petri dishes were placed upside down in opaque plastic trays lined with moistened paper towels. Furthermore, each tray was wrapped in a clear plastic bag that was sprayed on the inside with distilled water. The trays were incubated at temperatures between 16 and $18^{\circ} \mathrm{C}$ with a $12-\mathrm{h}$ photoperiod that was provided by cool white fluorescent lights in an incubator. For the first $24 \mathrm{~h}$ postinoculation, the trays were covered with black plastic to temporarily suppress the hypersensitive response and salicylic acid related defense responses (8). Seven days postinoculation, each droplet (spot) on each leaflet was scored as necrotic or nonnecrotic spot, as was the presence or absence of sporangia. In each experiment, 18 spots (from two petri dishes) were scored for each genotype. For each petri dish (containing three leaflets with nine spots), the percentage of spots with sporangia was considered the percent disease susceptibility (or percent disease severity [\%DS]) and the percentage of spots without sporangia was considered the percent disease resistance.

Field and GH screening experiments. The field and GH screening experiments with the same genotypes were conducted previously and reported elsewhere (4). The field and GH data from the previous study were used here for comparison with the detached-leaflet data.

Data analysis. For statistical purposes, each petri dish within each experiment, containing three leaflets of one genotype and a total of nine pathogen droplets, was considered one replication. For each genotype in each replication, the percentage of susceptibility (i.e., \%DS) was calculated as (number of necrotic spots with sporangia + number of nonnecrotic spots with sporangia)/(total number of spots inoculated) $\times 100$. Average $\%$ DS for each genotype in each experiment was calculated as the mean between the two replications (average over 18 spots). The final \%DS for each genotype was calculated as the average between the two experiments (average over 36 spots). The \%DS values within each experiment were subjected to analysis of variance (ANOVA), and mean comparisons were conducted using Fisher's protected least significant difference test (20). Pearson correlation coefficients $(r)$, based on \%DS across genotypes, were determined between replications within each detached-leaflet experiment and between the two detached-leaflet experiments. Pearson correlation coefficients $(r)$ were also determined between the detached-leaflet, field, and $\mathrm{GH}$ screening experiments. All statistical analyses were conducted using SPSS v. 22.0 (12) or R (18) software packages.

\section{Results}

There were wide variations in response to the pathogen among the 72 genotypes examined, from complete resistance to complete susceptibility (Table 1). Although the majority of genotypes were uniform across the two experiments, accessions LA1635, LA1923, LA2179, LA2335, PI 270444, PI 270447, and PI 272641 and control genotypes New Yorker and NC63EB showed varied responses in the two experiments (Table 1). Several factors could have caused this variation (see Discussion).

Across all tomato genotypes, there were significant $(P<0.001)$ correlations between replications within experiments (average $r=$ $0.75)$ and between the two detached-leaflet experiments $(r=0.72)$. Bartlett's test of homogeneity of variances indicated homogeneity of variances between the two experiments $\left(\lambda^{2}=2.7, P=0.1\right)$ and between the replications within experiments $\left(\lambda^{2}=0.094, P=0.75\right)$. Therefore, data from the two experiments were pooled and subjected to ANOVA, which indicated that there was no significant difference between the two experiments $(P=0.07)$ and replications nested within the experiments $(P=0.98)$. Both correlation analysis and ANOVA indicated that disease response in the detached-leaflet assay was generally consistent across replications and experiments.

Disease response (average \%DS) in the detached-leaflet assay was compared with disease responses in the field and GH experiments for the same genotypes, which were obtained from a previous study (4). Generally, there was a higher level of disease (\%DS) in the detachedleaflet assay (average $67.7 \pm 33.7$ ) compared with the field (average $60.6 \pm 36.5$ ) and GH experiments (average $49.3 \pm 28.7$ ), though the differences were not significant (Table 1). The higher level of disease in the detached-leaflet assay could be due to different factors, including higher concentration of inoculum and more favorable environmental conditions (e.g., higher relative humidity and better temperature) for disease progression. However, the average \%DS in the two detachedleaflet experiments was highly correlated $(P<0.001)$ with the average \%DS in the field $(r=0.82)$ and GH experiments $(r=0.84)$. These results indicated that, across tomato genotypes, response to LB in the detached-leaflet assay corresponded well to responses under field and $\mathrm{GH}$ conditions. In a previous study, using the same set of genotypes, we had determined that \%DS in different GH screening experiments were correlated with \%DS in different field screening experiments (average $r=0.72, P<0.001$ ) (4). In the present study, we determined that the correlation coefficient between the average \%DS in the field experiments and the average \%DS in the GH experiments (Table 1) was highly significant $(r=0.85, P<0.001)$, further indicating correspondences between field and GH responses to LB across tomato genotypes.

\section{Discussion}

The significance of LB and the potential for occurrence of new and more aggressive clonal lineages of $P$. infestans make identification of new sources of resistance and incorporation of resistance into commercial cultivars imperative objectives for many tomato-breeding programs. Attaining such objectives requires accurate, reliable, and efficient disease screening protocols. In a recent study, we evaluated the LB response of 72 tomato genotypes, including 67 S. pimpinellifolium 
Table 1. Late blight rating of tomato germplasm under different conditions

\begin{tabular}{|c|c|c|c|c|c|}
\hline \multirow[b]{2}{*}{ Genotype } & \multicolumn{3}{|c|}{ Detached leaflet (\%DS) } & \multicolumn{2}{|c|}{ Average $(\% \mathrm{DS})^{\mathrm{a}}$} \\
\hline & Exp. I & Exp. II & Average & Field & Greenhouse \\
\hline \multicolumn{6}{|c|}{ Solanum pimpinellifolium } \\
\hline LA0398 & $100.0 \pm 0.0$ & $100.0 \pm 0.0$ & $100.0 \pm 0.0$ & $94.5 \pm 9.7$ & $65.8 \pm 28.8$ \\
\hline LA0418 & $94.4 \pm 7.9$ & $100.0 \pm 0.0$ & $97.2 \pm 5.6$ & $100.0 \pm 0.0$ & $72.2 \pm 25.0$ \\
\hline LA0722 & $94.4 \pm 7.9$ & $100.0 \pm 0.0$ & $97.2 \pm 5.6$ & $93.5 \pm 9.1$ & $67.0 \pm 19.0$ \\
\hline LA1242 & $88.9 \pm 15.7$ & $100.0 \pm 0.0$ & $94.4 \pm 11.1$ & $90.0 \pm 13.5$ & $73.7 \pm 28.1$ \\
\hline LA1246 & $94.4 \pm 7.9$ & $100.0 \pm 0.0$ & $97.2 \pm 5.6$ & $92.5 \pm 15.0$ & $77.3 \pm 30.1$ \\
\hline LA1248 & $83.3 \pm 23.6$ & $100.0 \pm 0.0$ & $91.7 \pm 16.7$ & $80.0 \pm 40.0$ & $68.8 \pm 20.9$ \\
\hline LA1258 & $66.7 \pm 47.1$ & $100.0 \pm 0.0$ & $83.3 \pm 33.3$ & $87.5 \pm 25.0$ & $79.2 \pm 19.0$ \\
\hline LA1263 & $94.4 \pm 7.9$ & $100.0 \pm 0.0$ & $97.2 \pm 5.6$ & $82.0 \pm 34.7$ & $83.2 \pm 17.2$ \\
\hline LA1332 & $83.3 \pm 23.6$ & $100.0 \pm 0.0$ & $91.7 \pm 16.7$ & $80.0 \pm 40.0$ & $87.1 \pm 11.3$ \\
\hline LA1471 & $83.3 \pm 7.9$ & $94.4 \pm 7.9$ & $88.9 \pm 9.1$ & $87.5 \pm 25.0$ & $81.4 \pm 8.9$ \\
\hline LA1478 & $72.2 \pm 39.3$ & $94.4 \pm 7.9$ & $83.3 \pm 26.5$ & $87.5 \pm 25.0$ & $68.6 \pm 21.4$ \\
\hline LA1519 & $66.7 \pm 31.4$ & $100.0 \pm 0.0$ & $83.3 \pm 26.5$ & $72.5 \pm 41.7$ & $70.7 \pm 22.4$ \\
\hline LA1547 & $100.0 \pm 0.0$ & $100.0 \pm 0.0$ & $100.0 \pm 0.0$ & $84.0 \pm 5.4$ & $57.4 \pm 22.7$ \\
\hline LA1583 & $55.6 \pm 0.0$ & $77.8 \pm 15.7$ & $66.7 \pm 15.7$ & $95.0 \pm 10.0$ & $67.9 \pm 24.0$ \\
\hline LA1610 & $77.8 \pm 31.4$ & $100.0 \pm 0.0$ & $88.9 \pm 22.2$ & $50.0 \pm 24.5$ & $58.0 \pm 18.5$ \\
\hline LA1617 & $88.9 \pm 15.7$ & $94.4 \pm 7.9$ & $91.7 \pm 10.6$ & $100.0 \pm 0.0$ & $69.4 \pm 8.2$ \\
\hline LA 1635 & $33.3 \pm 15.7$ & $100.0 \pm 0.0$ & $66.7 \pm 39.5$ & $93.3 \pm 8.9$ & $86.1 \pm 10.9$ \\
\hline LA1684 & $66.7 \pm 0.0$ & $88.9 \pm 15.7$ & $77.8 \pm 15.7$ & $73.5 \pm 36.6$ & $67.4 \pm 19.9$ \\
\hline LA1688 & $77.8 \pm 0.0$ & $55.6 \pm 31.4$ & $66.7 \pm 22.2$ & $89.0 \pm 19.4$ & $52.0 \pm 24.2$ \\
\hline LA1719 & $83.3 \pm 7.9$ & $83.3 \pm 7.9$ & $83.3 \pm 6.4$ & $75.8 \pm 43.8$ & $56.1 \pm 22.0$ \\
\hline LA1728 & $88.9 \pm 15.7$ & $77.8 \pm 15.7$ & $83.3 \pm 14.3$ & $77.3 \pm 31.5$ & $43.0 \pm 20.2$ \\
\hline LA1923 & $50.0 \pm 70.7$ & $94.4 \pm 7.9$ & $72.2 \pm 48.4$ & $47.5 \pm 21.8$ & $37.2 \pm 9.4$ \\
\hline LA1924 & $77.8 \pm 15.7$ & $100.0 \pm 0.0$ & $88.9 \pm 15.7$ & $71.3 \pm 27.5$ & $61.1 \pm 20.9$ \\
\hline LA1933 & $77.8 \pm 15.7$ & $94.4 \pm 7.9$ & $86.1 \pm 14.0$ & $84.3 \pm 22.9$ & $68.3 \pm 13.0$ \\
\hline LA2093 & $83.3 \pm 23.6$ & $83.3 \pm 23.5$ & $83.3 \pm 19.2$ & $65.0 \pm 30.0$ & $56.1 \pm 18.8$ \\
\hline LA2096 & $61.1 \pm 23.6$ & $94.4 \pm 7.9$ & $77.8 \pm 24.0$ & $92.5 \pm 8.7$ & $68.9 \pm 23.2$ \\
\hline LA2097 & $100.0 \pm 0.0$ & $100.0 \pm 0.0$ & $100.0 \pm 0.0$ & $97.5 \pm 5.0$ & $66.9 \pm 22.4$ \\
\hline LA2102 & $83.3 \pm 23.6$ & $100.0 \pm 0.0$ & $91.7 \pm 16.7$ & $97.5 \pm 5.0$ & $50.2 \pm 25.0$ \\
\hline LA2112 & $83.3 \pm 7.9$ & $100.0 \pm 0.0$ & $91.7 \pm 10.6$ & $48.8 \pm 10.3$ & $48.0 \pm 11.6$ \\
\hline LA2179 & $33.3 \pm 47.1$ & $88.9 \pm 15.7$ & $61.1 \pm 43.0$ & 100.0 & $51.6 \pm 28.0$ \\
\hline LA2188 & $100.0 \pm 0.0$ & $100.0 \pm 0.0$ & $100.0 \pm 0.0$ & $87.0 \pm 11.9$ & $41.7 \pm 28.8$ \\
\hline LA2335 & $22.2 \pm 15.7$ & $100.0 \pm 0.0$ & $61.1 \pm 45.8$ & $28.8 \pm 10.3$ & $55.0 \pm 25.0$ \\
\hline LA2336 & $100.0 \pm 0.0$ & $100.0 \pm 0.0$ & $100.0 \pm 0.0$ & $86.5 \pm 24.4$ & $60.9 \pm 23.3$ \\
\hline LA2341 & $88.9 \pm 15.7$ & $100.0 \pm 0.0$ & $94.4 \pm 11.1$ & $87.0 \pm 24.7$ & $75.2 \pm 27.3$ \\
\hline LA2390 & $72.2 \pm 39.3$ & $55.6 \pm 15.7$ & $63.9 \pm 26.3$ & $85.0 \pm 30.0$ & $38.4 \pm 39.9$ \\
\hline LA2533 & $0.0 \pm 0.0$ & $0.0 \pm 0.0$ & $0.0 \pm 0.0$ & $0.0 \pm 0.0$ & $11.3 \pm 14.2$ \\
\hline LA2585 & $100.0 \pm 0.0$ & $100.0 \pm 0.0$ & $100.0 \pm 0.0$ & 60.0 & $68.8 \pm 36.3$ \\
\hline LA2646 & $94.4 \pm 7.9$ & $100.0 \pm 0.0$ & $97.2 \pm 5.6$ & $93.8 \pm 9.2$ & $48.1 \pm 30.0$ \\
\hline LA2839 & $77.8 \pm 31.4$ & $83.3 \pm 7.9$ & $80.6 \pm 19.0$ & $33.8 \pm 37.9$ & $19.1 \pm 11.9$ \\
\hline LA2866 & $66.7 \pm 47.1$ & $100.0 \pm 0.0$ & $83.3 \pm 33.3$ & $97.5 \pm 5.0$ & $71.7 \pm 20.8$ \\
\hline PI 126925 & $38.9 \pm 39.3$ & $44.4 \pm 31.4$ & $41.7 \pm 29.2$ & $75.0 \pm 23.5$ & $41.7 \pm 16.4$ \\
\hline PI 163245 & $61.1 \pm 39.3$ & $83.3 \pm 23.5$ & $72.2 \pm 29.4$ & $5.5 \pm 9.7$ & $12.2 \pm 7.1$ \\
\hline PI 224709 & $22.2 \pm 31.4$ & $66.7 \pm 31.4$ & $44.4 \pm 36.3$ & $65.0 \pm 12.2$ & $33.9 \pm 12.9$ \\
\hline PI 224710 & $5.6 \pm 7.9$ & $0.0 \pm 0.0$ & $2.8 \pm 5.6$ & $15.0 \pm 10.0$ & $10.7 \pm 3.7$ \\
\hline PI 263589(O) & $83.3 \pm 23.6$ & $100.0 \pm 0.0$ & $91.7 \pm 16.7$ & 100.0 & $66.4 \pm 31.0$ \\
\hline PI 270439 & $38.9 \pm 7.9$ & $0.0 \pm 0.0$ & $19.4 \pm 22.9$ & $3.0 \pm 4.7$ & $4.1 \pm 3.1$ \\
\hline PI 270440 & $0.0 \pm 0.0$ & $5.6 \pm 7.8$ & $2.8 \pm 5.6$ & $8.0 \pm 4.0$ & $13.6 \pm 6.3$ \\
\hline PI 270441(O) & $16.7 \pm 23.6$ & $0.0 \pm 0.0$ & $8.3 \pm 16.7$ & $0.5 \pm 0.4$ & $3.1 \pm 3.1$ \\
\hline PI 270442(O) & $11.1 \pm 15.7$ & $0.0 \pm 0.0$ & $5.6 \pm 11.1$ & $2.9 \pm 4.8$ & $1.5 \pm 1.8$ \\
\hline PI 270443 & $0.0 \pm 0.0$ & $0.0 \pm 0.0$ & $0.0 \pm 0.0$ & $2.8 \pm 4.9$ & $3.7 \pm 3.4$ \\
\hline PI 270444 & $50.0 \pm 23.6$ & $0.0 \pm 0.0$ & $25.0 \pm 31.9$ & $3.5 \pm 4.7$ & $4.2 \pm 6.3$ \\
\hline PI 270445 & $33.3 \pm 47.1$ & $22.2 \pm 31.4$ & $27.8 \pm 33.3$ & $0.3 \pm 0.5$ & $4.1 \pm 2.1$ \\
\hline PI 270446 & $44.4 \pm 0.0$ & $50.0 \pm 7.9$ & $47.2 \pm 5.6$ & $1.0 \pm 0.8$ & $4.2 \pm 3.0$ \\
\hline PI 270447 & $88.9 \pm 15.7$ & $0.0 \pm 0.0$ & $44.4 \pm 52.1$ & $0.6 \pm 0.5$ & $5.4 \pm 4.7$ \\
\hline PI 270448 & $38.9 \pm 7.9$ & $0.0 \pm 0.0$ & $19.4 \pm 22.9$ & $0.1 \pm 0.3$ & $4.0 \pm 5.1$ \\
\hline PI 270449 & $16.7 \pm 23.6$ & $0.0 \pm 0.0$ & $8.3 \pm 16.7$ & $0.1 \pm 0.3$ & $2.9 \pm 3.6$ \\
\hline PI 270450 & $22.2 \pm 15.7$ & $0.0 \pm 0.0$ & $11.1 \pm 15.7$ & $3.0 \pm 4.7$ & $3.1 \pm 2.9$ \\
\hline PI 270451 & $33.3 \pm 15.7$ & $16.7 \pm 23.6$ & $25.0 \pm 19.0$ & $0.3 \pm 0.5$ & $2.6 \pm 1.9$ \\
\hline PI 270452 & $94.4 \pm 7.9$ & $100.0 \pm 0.0$ & $97.2 \pm 5.6$ & $66.3 \pm 14.4$ & $79.1 \pm 25.8$ \\
\hline PI 270453 & $94.4 \pm 7.9$ & $100.0 \pm 0.0$ & $97.2 \pm 5.6$ & $67.5 \pm 5.0$ & $68.0 \pm 26.3$ \\
\hline PI 272641 & $16.7 \pm 23.6$ & $100.0 \pm 0.0$ & $58.3 \pm 50.0$ & $56.3 \pm 7.5$ & $73.9 \pm 17.8$ \\
\hline PI 272652 & $94.4 \pm 7.9$ & $100.0 \pm 0.0$ & $97.2 \pm 5.6$ & $76.3 \pm 17.5$ & $76.3 \pm 25.9$ \\
\hline & & & & & on next page) \\
\hline
\end{tabular}

\footnotetext{
a Field (average of two experiments) and greenhouse (average of four experiments) data were taken from a previous study (4).

b $\mathrm{SD}=$ standard deviation.

c Least significant difference.
} 
Table 1. (continued from preceding page)

\begin{tabular}{|c|c|c|c|c|c|}
\hline \multirow[b]{2}{*}{ Genotype } & \multicolumn{3}{|c|}{ Detached leaflet (\%DS) } & \multicolumn{2}{|c|}{ Average $(\% \mathrm{DS})^{\mathrm{a}}$} \\
\hline & Exp. I & Exp. II & Average & Field & Greenhouse \\
\hline PI 272656 & $100.0 \pm 0.0$ & $100.0 \pm 0.0$ & $100.0 \pm 0.0$ & $84.3 \pm 11.1$ & $74.1 \pm 29.6$ \\
\hline PI 274174 & $88.9 \pm 15.7$ & $100.0 \pm 0.0$ & $94.4 \pm 11.1$ & $95.3 \pm 3.5$ & $72.6 \pm 32.4$ \\
\hline PI 312131 & $88.9 \pm 15.7$ & $100.0 \pm 0.0$ & $94.4 \pm 11.1$ & $76.3 \pm 6.3$ & $75.6 \pm 18.6$ \\
\hline PI 438897 & $88.9 \pm 15.7$ & $100.0 \pm 0.0$ & $94.4 \pm 11.1$ & $82.3 \pm 12.1$ & $67.4 \pm 35.5$ \\
\hline PI438898 & $50.0 \pm 23.6$ & $100.0 \pm 0.0$ & $75.0 \pm 31.9$ & 20.0 & $83.4 \pm 22.2$ \\
\hline \multicolumn{6}{|c|}{ Controls (S. lycopersicum) } \\
\hline NC84173 & $72.2 \pm 7.9$ & $100.0 \pm 0.0$ & $86.1 \pm 16.7$ & $\ldots$ & $83.9 \pm 11.5$ \\
\hline New Yorker & $38.9 \pm 39.3$ & $100.0 \pm 0.0$ & $69.4 \pm 41.9$ & $\ldots$ & $82.9 \pm 21.3$ \\
\hline NC63EB & $66.7 \pm 47.1$ & $5.6 \pm 7.9$ & $36.1 \pm 44.8$ & $\ldots$ & $18.9 \pm 11.9$ \\
\hline NC870 & $0.0 \pm 0.0$ & $0.0 \pm 0.0$ & $0.0 \pm 0.0$ & $\ldots$ & $13.7 \pm 6.9$ \\
\hline $\mathrm{NC} 03220$ & $0.0 \pm 0.0$ & $0.0 \pm 0.0$ & $0.0 \pm 0.0$ & $\ldots$ & $6.2 \pm 9.4$ \\
\hline Mean $\pm \mathrm{SD}^{\mathrm{b}}$ & $63.4 \pm 31.7$ & $71.6 \pm 40.4$ & $67.5 \pm 33.5$ & $60.6 \pm 36.5$ & $49.3 \pm 28.7$ \\
\hline $\operatorname{LSD}(0.05)^{\mathrm{c}}$ & 46.1 & 20.4 & 31.4 & 26.8 & 18.5 \\
\hline
\end{tabular}

wild accessions and 5 S. lycopersicum control lines, under field and GH conditions (4). Across genotypes, there were significant correlations (average $r=0.72$ ) between field and GH experiments in disease responses, suggesting a good correspondence between field and GH screening methods and that either method could be used reliably for evaluating tomato response to LB. However, both field and GH disease screenings using whole plants can be time and labor intensive and may require a large amount of space, facilities, and pathogen inoculum. Further, field evaluation may only be conducted during the growing season, often once a year, and may be prone to other uncontrollable environmental and biological factors.

The purpose of the present study was to determine whether a detached-leaflet assay could be used reliably to screen tomato for response to LB, and whether resistance at this level reflects resistance at the whole-plant level under field or GH conditions. We evaluated LB response of the same 72 tomato genotypes, which varied in their response to LB from very resistant to highly susceptible (4), in two replicated detached-leaflet experiments and compared their \%DS scores with those obtained under field and GH conditions. The presence of significant correlations between replications within experiments (average $r=0.75, P<0.001$ ) and between the two experiments (average $r=$ $0.72, P<0.001)$ suggests reasonable consistency of results obtained from detached-leaflet screening experiments. Although the majority of genotypes were uniform in their response to P. infestans in the two experiments, several genotypes exhibited variation from experiment to experiment (Table 1). Such variation could be caused by different nongenetic factors, including physiological age and size of the leaflets, spore concentration in the inoculum droplets (potential disease escape), microenvironmental variation from plate to plate, and variation in the position of plates in the tray and in the incubator. These are considered potential limitations of this screening technique, which should be considered when conducting detached-leaflet assays. However, the magnitudes of the correlation coefficients observed in this study were similar to those previously reported between different field or different GH LB experiments $(2,4,15,21)$, suggesting that the congruency among different detached-leaflet experiments is similar to that observed among different field or $\mathrm{GH}$ experiments. Replicating experiments and minimizing nongenetic variation should improve the reliability of the results in detached-leaflet assays.

The presence of significant correlations between results from the detached-leaflet assay and those from field $(r=0.82)$ and GH assays $(r=0.84)$ suggests good correspondences among these screening methods, and that they produce similar results. The magnitudes of these correlation coefficients are particularly important in light of evaluation of a diverse set of tomato accessions with a wide range of resistance or susceptibility to LB. Therefore, the detached-leaflet assay can be used confidently as an alternative or additional screening method for distinguishing among LB-resistant and LB-susceptible tomato genotypes. This is in agreement with the results reported by Vleeshouwers et al. (24), who examined reactions of different potato
Solanum spp. to LB under field conditions and compared them with their responses to LB using detached leaves under growth chamber conditions. Similarly, Goth and Keane (7) did not find significant differences in reactions to isolates of $P$.infestans on detached and intact leaves of two potato cultivars and one tomato cultivar. Michalska et al. (15) examined LB response of over two dozen cultivars and breeding lines of potato and reported that a detached-leaflet assay could be useful for screening of potato for LB resistance. In contrast, laboratory assays of detached leaflets, leaf disks, and stem cuttings of potato genotypes were reported to be less reliable than GH assays with whole plants for overall ratings of partial resistance, though laboratory assays were considered useful for measuring specific components of resistance (2). In the latter study, variations were observed among the laboratory assays.

Practical considerations, such as low cost and minimal facility requirements for conducting detached-leaflet experiments, the need for leaves or leaflets as opposed to whole plants, the small amount of inoculum needed, minimal risk of pathogen escaping into commercial growing environments, and being fast and nondestructive make the detached-leaflet assay attractive, in particular for largescale screening of tomato breeding populations. The detachedleaflet assay may also be useful for screening of breeding material to reduce the number of entries in field trials $(2,15)$. Further, a researcher wishing to evaluate response to $\mathrm{LB}$ with a new isolate or unravel resistance mechanisms may need to conduct experiments under confined and controlled conditions, such as in a growth chamber. The detached-leaflet assay may also be used in parallel with whole-plant evaluations to provide further support to results obtained from other screening methods.

To our knowledge, this is the first time a detached-leaflet method for evaluating tomato response to LB and its comparison with field and GH screening assays is reported. The consistency of results within and between detached-leaflet experiments and the moderately high correlations between disease response in the detached-leaflet assay and those under field and GH conditions indicate that this screening assay could be used with reasonable confidence to distinguish among LB-resistant and LB-susceptible tomato plants. Based on the genotypes evaluated here, it appears that the detached-leaflet assay is as reliable as the field or GH screening assays. However, it has the advantages of simplicity and minimal requirement of space, facility, and pathogen inoculum. Further, the detached-leaflet assay can be highly efficient and more practical when screening large breeding populations.

\section{Acknowledgments}

We thank G. Moorman and B. Gugino for reviewing this manuscript before submission and making constructive comments and suggestions, H. Merk for reviewing an earlier version of this manuscript and making useful comments, Penn State staff and students who helped with the disease screening experiments, S.-H. Kim of the Pennsylvania Department of Agriculture for providing original isolates of $P$. infestans, R. Gardner of the NC State University for providing seed of several 
control breeding lines, and the USDA PGRU and TGRC for providing the original seed of the wild accessions. This research was supported, in part, by Agricultural Research Funds administered by the Pennsylvania Department of Agriculture, the Pennsylvania Vegetable Marketing and Research Program, and the College of Agricultural Sciences at the Pennsylvania State University.

\section{Literature Cited}

1. Birhman, R. K., and Singh, B. P. 1995. Path-coefficient analyses and genetic parameters of the components of field resistance of potatoes to late blight. Ann. Appl. Biol. 127:353-362.

2. Dorrance, A. E., and Inglis, D. A. 1997. Assessment of greenhouse and laboratory screening methods for evaluating potato foliage for resistance to late blight. Plant Dis. 81:1206-1213.

3. Foolad, M. R., Merk, H. L., and Ashrafi, H. 2008. Genetics, genomics and breeding of late blight and early blight resistance in tomato. Crit. Rev. Plant Sci. 27:75-107.

4. Foolad, M. R., Sullenberger, M. T., Ohlson, E. W., and Gugino, B. C. 2014. Response of accessions within tomato wild species, Solanum pimpinellifolium to late blight. Plant Breed. 133:401-411.

5. Gardner, R. G., and Panthee, D. R. 2010. NC 1 CELBR and NC 2 CELBR: Early blight and late blight resistant fresh market tomato breeding lines. HortScience 45:975-976.

6. Gardner, R. G., and Panthee, D. R. 2010. 'Plum Regal' Fresh-market plum tomato hybrid and its parents, NC 25P and NC 30P. HortScience 45:824-825.

7. Goth, R. W. 1997. A detached-leaf method to evaluate late blight resistance in potato and tomato. Am. Potato J. 74:347-352.

8. Griebel, T., and Zeier, J. 2008. Light regulation and daytime dependency of inducible plant defenses in Arabidopsis: Phytochrome signaling controls systemic acquired resistance rather than local defense. Plant Physiol. 147: 790-801

9. Harrison, J., Barker, H., Lowe, R., and Rees, E. 1990. Estimation of amounts of Phytophthora infestans mycelium in leaf tissue by enzyme-linked immunosorbent assay. Plant Pathol. 39:274-277.

10. Hodgson, W. 1961. Laboratory testing of the potato for partial resistance to Phytophthora infestans. Am. Potato J. 38:259-264.
11. Hodgson, W. 1962. Studies on the nature of partial resistance in the potato to Phytophthora infestans. Am. Potato J. 39:8-13.

12. IBM-Corp. 2014. IBM SPSS Statistics for Windows, Version 22.0, Released 2013. IBM Corp., Armonk, NY.

13. Lapwood, D. 1961. Laboratory assessments of the susceptibility of potato haulm to blight (Phytophthora infestans). Eur. Potato J. 4:117-127.

14. Malcolmson, J. F. 1969. Factors involved in resistance to blight (Phytophthora infestans (Mont.) de Bary) in potatoes and assessment of resistance using detached leaves. Ann. Appl. Biol. 64:461-468.

15. Michalska, A. M., Zimnoch-Guzowska, E., Sobkowiak, S., and Plich, J. 2011 Resistance of potato to stem infection by Phytophthora infestans and a comparison to detached leaflet and field resistance assessments. Am. J. Potato Res. 88:367-373.

16. Nowicki, M., Foolad, M. R., Nowakowska, M., and Kozik, E. U. 2012. Potato and tomato late blight caused by Phytophthora infestans: An overview of pathology and resistance breeding. Plant Dis. 96:4-17.

17. Panthee, D. R., and Gardner, R. G. 2010. 'Mountain Merit': A late blightresistant large-fruited tomato hybrid. HortScience 45:1547-1548.

18. R Core Team. 2014. R: A Language and Environment for Statistical Computing R Foundation for Statistical Computing, Vienna. http://www.R-project.org/

19. Singh, B. P., and Birhman, R. K. 1994. Laboratory estimation of field resistance of potato to late blight. J. Phytopathol. 140:71-76.

20. Steel, R. G. D., Torrie, J. H., and Dickey, D. A. 1997. Principles and Procedures of Statistics; a Biometrical Approach. McGraw-Hill, New York.

21. Stewart, H. E., Flavelle, P. H., McCalmont, D. C., and Wastie, R. L. 1983. Correlation between glasshouse and field tests for resistance to foliage blight caused by Phytophthora infestans. Potato Res. 26:41-48.

22. Toxopeus, H. J. 1958. Some notes on the relations between field resistance to Phytophthora infestans in leaves and tubers and ripening time in Solanum tuberosum subsp. tuberosum. Euphytica 7:123-130.

23. Umaerus, V., and Lihnell, D. 1976. A laboratory method for measuring the degree of attack by Phytophthora infestans. Potato Res. 19:91-107.

24. Vleeshouwers, V. G. A. A., van Dooijeweert, W., Keijzer, L. C. P., Sijpkes, L. Govers, F., and Colon, L. T. 1999. A laboratory assay for Phytophthora infestans resistance in various Solanum species reflects the field situation. Eur. J. Plant Pathol. 105:241-250. 